\title{
Impact of a guideline-based best practice alert on pneumococcal vaccination rates in adults in a primary care setting
}

Carrie McAdam-Marx ${ }^{1,2^{*}}$, Casey Tak ${ }^{3}$, Tanaz Petigara ${ }^{4}$, Nathan W. Jones ${ }^{5}$, Minkyoung Yoo ${ }^{1,6}$, Melissa Struwe Briley ${ }^{7}$, Karen Gunning ${ }^{1,8}$ and Lisa Gren ${ }^{5}$

\begin{abstract}
Background: Despite the high burden of pneumococcal disease, pneumococcal vaccine coverage continues to fall short of Healthy People 2020 goals. A quasi-experimental design was used to investigate the impact of pneumococcal-specific best-practice alerts (BPAs) with and without workflow redesign compared to health maintenance notifications only, on pneumococcal vaccination rates in at-risk and high-risk adults, and on series completion in immunocompetent adults aged $65+$ years.
\end{abstract}

Methods: This retrospective study used electronic health record and administrative data to identify pneumococcal vaccinations using cross sectional and historical cohorts of adults age 19+ years from 2013 to 2017 who attended clinics associated with the University of Utah Health. Difference-in-differences (DD) analyses was used to assess the impact of interventions across three observation periods (Baseline, Interim, and Follow Up). Adherence to the 2dose vaccination schedule in older adults was measured through a longitudinal analysis.

Results: In DD analyses, implementing both workflow redesign and the BPA raised the vaccination rate by 8 percentage points $(\mathrm{pp})(P<0.001)$ and implementing the BPA only raised the rate by $7 \mathrm{pp}$. $(P<0.001)$ among at-risk adults age 19-64 years, relative to implementing health maintenance notifications (i.e., usual care) only in comparison clinics. In high-risk adults age 19-64 years, the BPA with or without workflow redesign did not significantly affect vaccination rates from baseline to follow up relative to health maintenance notifications. Per DD analyses, the effect of the BPA was mixed in immunocompetent and immunocompromised adults age 65+ years. However, immunocompetent older adults attending a clinic that implemented the BPA plus health maintenance notifications and workflow redesign (all 3 interventions) had 1.94 times higher odds (Odds ratio (OR) 1.94; $P=$ $0.0003,95 \% \mathrm{Cl} 1.24,3.01)$ to receive the second pneumococcal dose than patients attending a usual practice clinic (i.e., no intervention).

Conclusions: A pneumococcal BPA tool that reflects current guidelines implemented with and without workflow redesign improved vaccination rates for at-risk adults age 19-64 years and increased the likelihood of adults aged $65+$ to complete the recommended 2-dose series. However, in other adult patient groups, the BPA was not consistently associated with improvements in pneumococcal vaccination rates.

Keywords: Pneumonia, pneumococcal, Pneumococcal infections, Risk factors, Immunocompromised patients, Health resources, Health care costs

\footnotetext{
* Correspondence: cmcadammarx@uams.edu

'Department of Pharmacotherapy, University of Utah, Salt Lake City, UT, USA

${ }^{2}$ Division of Pharmaceutical Evaluation \& Policy, University of Arkansas for

Medical Sciences, 4301 W. Markham St, Little Rock, AR 72205, USA

Full list of author information is available at the end of the article
}

(c) The Author(s). 2019 Open Access This article is distributed under the terms of the Creative Commons Attribution 4.0 International License (http://creativecommons.org/licenses/by/4.0/), which permits unrestricted use, distribution, and reproduction in any medium, provided you give appropriate credit to the original author(s) and the source, provide a link to the Creative Commons license, and indicate if changes were made. The Creative Commons Public Domain Dedication waiver (http://creativecommons.org/publicdomain/zero/1.0/) applies to the data made available in this article, unless otherwise stated. 


\section{Background}

Pneumococcal disease is a leading cause of morbidity and mortality, especially among immunocompromised individuals and older adults. [1] Annual total direct and indirect costs associated with pneumococcal disease in the US are estimated to be $\$ 5.1$ billion. [1] Guidelines for the use of approved pneumococcal vaccines in the US have been developed by the Advisory Committee on Immunization Practices (ACIP). [2, 3]

Since 1997, the ACIP has recommended a single dose of the 23-valent pneumococcal polysaccharide vaccine (PPSV23) for adults aged 19-64 years with certain chronic conditions (such as chronic heart disease, chronic lung disease and diabetes) that put them at risk for pneumococcal infections and/or poor outcomes from an infection (hereafter referred to as at-risk adults). [2] In 2012, the 13-valent pneumococcal conjugate vaccine (PCV13) followed by PPSV23 vaccination 8 weeks later was recommended for vaccine-naïve adults aged 19+ years who may be immunocompromised due to a condition or its treatment such as chronic renal failure, HIV and cancer (hereafter referred to as high-risk adults). [2] Both PCV13 and PPSV23 were recommended for adults aged $65+$ in 2014 with updates to recommendations regarding timing of the second dose in 2015 [3] (Additional file 1: Table S1).

Despite the high burden of pneumococcal disease, pneumococcal vaccine coverage continues to fall short of Healthy People 2020 goals $(60 \%$ among high-risk adults aged 19-64 years and 90\% among persons aged 65+). [4] In 2015 in the US, only $23 \%$ of at-risk and high-risk adults aged $19-64$ years and $64 \%$ of adults aged $65+$ reported ever receiving a pneumococcal vaccination in their lifetime. [5]

Clinical decision support tools can support health care providers to identify patients who are eligible for recommended preventive services. [6] One such tool is an electronic medical record-based clinical alert system, or Best Practice Alert (BPA). A BPA triggers a patient-specific alert when a set of criteria is met, providing guidance regarding specific health needs that can be considered during the visit. For vaccinations, BPAs can assist in identifying appropriate patients, update a patient's vaccination history in the electronic medical record if unavailable, and document reasons for vaccination refusal. [6-8]

Electronic medical record-based alerts or reminders targeted towards healthcare providers have been shown to be effective in improving vaccine coverage and documentation rates in children, adolescents and adults. Following BPA implementation in one academic institution and one community practice clinic, the influenza and pneumococcal vaccination rates increased from 47 to $65 \%$ and 19 to $41 \%$ ( $P<0.001$ for both $)$ respectively. [7]
Similarly, an evaluation of an electronic medical recordbased vaccination reminder at 4 community pediatric clinics found that the number of eligible children who remained unvaccinated was significantly lower at all clinics when the reminder was switched on compared to when the reminder was switched off (adjusted RR 0.90 [0.83-0.98]). [8]

In 2016, the University of Utah Health (U of U Health) developed clinical decision support tools to support the implementation of ACIP pneumococcal vaccination guidelines in all age and risk groups.

Two tools were developed - a passive health maintenance notification and a proactive BPA or pop-up message. The health maintenance screen, which is part of the electronic medical record and is implemented across all clinics, alerts staff to preventative care needs, including vaccinations. Typically, nursing staff pull up and review the health maintenance screen upon rooming the patient. For pneumococcal vaccinations, the notification prompts the staff to discuss the vaccination with patients and includes links to vaccination guidelines and order templates. The pneumococcal BPA sends a pop-up message to the healthcare provider at the time of order entry (for any reason) stating the vaccination need for that patient. The BPA serves as a follow-through reminder to the health maintenance notification, and the decision to implement the tool is made at the clinic level.

Prior to the release of the pneumococcal clinical decision support tools (in 2015), several clinics that also implemented the BPA evaluated and modified their pneumococcal vaccination workflows to fully integrate the BPA as part of their quality improvement efforts. The workflow redesign involved mapping current activities, listing all activities and who performs them, identifying opportunities to improve or streamline processes, and incorporation of the desired improvements, to ultimately achieve the desired process and/or patient outcomes.

The purpose of this study was to assess the impact of health maintenance notifications and pneumococcalspecific BPAs with and without workflow redesign, on pneumococcal vaccination rates in at-risk and high-risk adults (age 19-64 years and age 65+ years) attending $U$ of $U$ Health community-based clinics, and on series completion in immunocompetent adults aged $65+$ years.

\section{Methods}

\section{Study design and data sources}

This retrospective study used electronic health record and administrative data to identify pneumococcal vaccinations using cross sectional and historical cohorts from 2013 to 2017 who attended Family Medicine (FM) and Internal Medicine (IM) clinics associated with the $\mathrm{U}$ of U Health. 
The primary data source for assessing vaccination rates was the $U$ of $U$ Health Enterprise Data Warehouse (EDW). The EDW contains administrative and electronic medical record data for 1.4 million patients treated at the $U$ of $U$ Health from 1990 to present day, including vaccination history from the Utah State Immunization Information System (USIIS). Diagnosis codes and medication orders in the EDW were used to identify adults recommended for pneumococcal vaccination based on age and presence of at-risk or high-risk conditions. Vaccine administration and vaccination history were identified through electronic medical recordbased care delivery and billing codes (CPT) for vaccines administered by a $U$ of $U$ Health provider. These data were integrated with the EDW by the implementation of a system-wide, real-time interface in April 2016.

Prior to the launch of the revised clinical decision support tools, a subgroup of four FM clinics undertook a process mapping and redesign exercise as a Quality Improvement initiative. These clinics reviewed their pneumococcal vaccination workflow, identified the most appropriate point of clinical decision support pneumococcal report generation, then proposed and implemented redesigned workflows to integrate the existing clinical decision support pneumococcal reports into routine practice during the first half of the 2015 calendar year. The redesigns were clinic specific, but in general they identified processes to identify patients in need of vaccination prior to the visit, confirm a patient's vaccination history during the visit, and document vaccine administration details including patient refusal or receipt of vaccination elsewhere. For immunocompromised individuals and older adults (aged 65+) who required sequential PCV13-PPV23 vaccination, an additional step of scheduling the next appointment was incorporated into the workflow.

A pre-test post-test design with a non-equivalent comparison group was used to evaluate the impact of health maintenance notifications, BPAs and clinical workflow redesign on vaccination rates. Three clinic groups were included in the analysis. Four FM Clinics that received the BPA tool, health maintenance notifications, and also implemented workflow redesign to integrate the tools at the appropriate point of care (FM Group A); 7 FM Clinics that received the BPA tool and health maintenance notifications, but did not undertake workflow redesign (FM Group B); and 10 IM Clinics that received health maintenance notifications but did not receive the BPA tool and did not implement any workflow redesign (IM Group C). The IM clinics served as the nonequivalent comparison group.

\section{Study population}

The study population consisted of adults (aged 19+ years) receiving care at a $U$ of $U$ Health FM or IM clinic who met at least 1 of the ACIP criteria for pneumococcal vaccination. At-risk or high-risk adults were aged 19-64 years, while immunocompetent and immunocompromised adults were aged $65+$.

\section{Measures}

Pneumococcal vaccination rates were determined for 3 observation periods (Baseline, Interim, and Follow-up) at all clinics included in the study (Table 1) based on vaccinations given in clinic and documentation of doses administered at other sites of care per patient recall or USIIS. Vaccination rates were calculated in patients who were eligible for pneumococcal vaccination (at-risk/highrisk adults or adults aged 65+), and who had a history of care with one of the study clinics (at least 1 office visit within the 12 months prior to the observation period). Vaccination rates measured whether patients in FM and comparison IM clinics were up-to-date with pneumococcal vaccination according to ACIP guidelines in place at the end of each observation period. For at-risk adults age 19-64 years old, vaccination rates measured whether adults were up-to-date with PPSV23 vaccination by the end of the observation period. For high-risk adults age 19-64 years old, vaccination rates measured whether adults were up-to-date with the appropriate number of doses (PCV13, PPSV23 or both) by the end of the observation period.

The baseline period (August 1, 2013 - July 31, 2014) represents the period of time prior to BPA, health maintenance notifications, and workflow improvements. The interim period (May 1, 2015 - April 30, 2016) represents

Table 1 Interventions implemented during observation periods by clinic group: vaccination rate analyses

\begin{tabular}{|c|c|c|c|c|}
\hline $\begin{array}{l}\text { Observation } \\
\text { period }\end{array}$ & Time Frame & Family Medicine Clinics (FM Group A) & Family Medicine Clinics (FM Group B) & $\begin{array}{l}\text { Internal Medicine Clinics } \\
\text { (IM Group C) }\end{array}$ \\
\hline Baseline & $\begin{array}{l}\text { August 1,2013 - July } \\
31,2014\end{array}$ & Current Practice & Current Practice & Current Practice \\
\hline Interim & $\begin{array}{l}\text { May 1, } 2015 \text { - April } \\
30,2016\end{array}$ & Clinical workflow redesign & Current Practice & Current Practice \\
\hline Follow-up & $\begin{array}{l}\text { May 1, } 2016-\text { Jul 31, } \\
2017\end{array}$ & $\begin{array}{l}\text { Workflow redesign plus BPA tool \& Health } \\
\text { Maintenance Notification }\end{array}$ & $\begin{array}{l}\text { Best practice alert tool \& Health } \\
\text { Maintenance Notification }\end{array}$ & $\begin{array}{l}\text { Health Maintenance } \\
\text { Notification }\end{array}$ \\
\hline
\end{tabular}


Table 2 Interventions implemented at the time of first and second pneumococcal dose: series completion analysis

\begin{tabular}{|c|c|c|c|}
\hline Clinic Group & Timeframe for Second Dose & Intervention & Exposure Group \\
\hline \multicolumn{4}{|c|}{ June 2014-April 2016: Dose 1 timeframe } \\
\hline$B, C$ & June 2014-April 2016 & Current practice & 1 \\
\hline A & June 2014-April 2015 & Current practice & 1 \\
\hline A & May 2015-April 2016 & Workflow redesign only & 2 \\
\hline \multicolumn{4}{|c|}{ Dec 2014 - July 2017: Dose 2 timeframe } \\
\hline$B, C$ & May 2015-April 2016 & Current practice & 1 \\
\hline A & May 2015-April 2016 & Workflow redesign only & 2 \\
\hline C & May 2016-July 2017 & Health maintenance only & 3 \\
\hline B & May 2016-July 2017 & BPA \& health maintenance & 4 \\
\hline A & May 2016-July 2017 & BPA \& health maintenance + Workflow redesign & 5 \\
\hline
\end{tabular}

the period of time during which 4 of the 11 FM Clinics undertook a redesign of pneumococcal vaccination workflow processes but before the BPA tool and health maintenance notifications were implemented. The follow-up period (May 1, 2016 - July 31, 2017) represents the time during which the BPA tool was rolled out to all FM Clinics, and the health maintenance notification rolled out to both FM and IM clinics.

Adherence to the 2-dose vaccination schedule in older adults was measured through a longitudinal analysis of immunocompetent adults aged $65+$ who received the first pneumococcal vaccination between June 1, 2014 and April 30, 2016, and who were due to receive the second vaccination between December 1, 2014 and July 31, 2017. The proportion of patients who completed the series was compared by clinical decision support tool and workflow redesign status at the time the patient's second dose was due: [1] current practice (no intervention); [2] workflow redesign only; [3] health maintenance notification only; [4] BPA and health maintenance notification; and [5] workflow redesign and BPA and health maintenance notification (Table 2).

\section{Statistical analysis}

Descriptive statistics (count, percentage, mean, standard deviation, 95\% confidence intervals) were used to compare patient characteristics between clinic groups for each observation period.

For each observation period (Baseline, Interim, and Follow-up), the vaccination rate was identified for the study clinic populations and was defined as:
To assess vaccination rates while minimizing confounding from the non-random sample, the differencein-differences (DD) method with a block bootstrap was used, with exposure defined as the intervention in place at the patient's assigned clinic at the end of each observation period. The DD approach controls for unobserved differences between clinics that are related to both the intervention and vaccination rates, while controlling for trends over time that may affect vaccination rates across all clinics. $[9,10]$ Demographic and clinical covariates were included in the regression models to control for differences in the composition of patient groups that could lead to confounding.

Mixed effects logistic regression under the generalized linear mixed models (GLMMs) was used to identify associations between intervention exposure at the time the second dose was due and series completion in older adults, controlling for potential confounders. Mixed effects logistic regression accounts for the dependence of observations because of the clustering of patients within clinics. Due to the hierarchical structure of the data, three level mixed effects logistic regression was also performed to account for possible clustering both within clinics and time period.

\section{Results}

Vaccination rates in at-risk adults age 19-64 years

Of 18,851 individuals age 19-64 years who met study inclusion criteria in the baseline period, 16,193 (86\%) were considered at-risk (immunocompetent with chronic medical conditions), with $84 \%$ (18,423 out of 21,872

Number of patients whose vaccination status was up-to-date according to ACIP guidelines by the end of the observation period Number of patients recommended for pneumococcal vaccination according to ACIP guidelines by the end of the observation period 
patients) and $86 \%$ (21,498 out of 25,110 patients) of patients identified as at-risk in the interim and follow-up periods, respectively.

Age and sex of at-risk adults age 19-64 for each observation period are provided in Table 3. Characteristics of at-risk patients age 19-64 years are presented overall and by clinic for each observation period in the Additional file 1: Tables S2-S4. Overall, patient age, sex, and race were similar across the 3 time periods; patient mean age was 44 years, $55 \%$ of patients were female, and nearly three-quarters were Caucasian. The proportion of patients who were commercially insured ranged from $57 \%$ in the first observation period to $67 \%$ in the third observation period. Cigarette smoking (40 to 45\%), chronic lung disease (33 to 38\%), and diabetes (28 to $29 \%)$ were the 3 most common at-risk conditions across the observation periods.

In each observation period, patients in the IM Group C clinics were 4-5 years older on average than patients in FM A and B clinics. IM Group C clinics also had a lower proportion of female patients and cigarette smokers compared to FM A and B clinics, and a higher proportion of patients with diabetes. FM Group B clinics had a higher proportion of Hispanic patients and a lower proportion of commercially insured patients than FM Group A or IM Group C clinics in each observation period.

Vaccination rates in the at-risk 19-64 years old population measured whether adults were up-to-date with PPSV23 vaccination by the end of the observation period. The unadjusted vaccination rate in this group overall improved from $25.3 \%$ in the baseline period to 29.7 and $36.1 \%$ in the interim and follow up periods respectively $(P<0.001)$ (Additional file 2: Figure S1). While the vaccination rate improved in each clinic over the 3 observation periods, the rate was significantly lower in the intervention clinics (FM Group A and FM Group B) than the IM
Group C clinics in the baseline and interim periods. No difference was detected during the follow-up period among the 3 clinics.

DD analyses showed that the clinics with the BPA, with or without workflow redesign (FM Group A and FM Group B), had greater improvement in vaccination rates from baseline to follow up relative to clinics with health maintenance notifications only (IM Group C clinics), controlling for demographic and clinical characteristics and underlying trends. Implementing both workflow redesign and the BPA (FM Group A) raised the vaccination rate by 8 percentage points $(P<0.001)$, and implementing the BPA only (FM Group B) raised the rate by 7 percentage points $(P<0.001)$, relative to implementing health maintenance notifications only (IM Group C) (Additional file 3: Figure S2).

\section{Vaccination rates in high-risk adults age 19-64 years}

Of 18,851 patients age 19-64 years who met study inclusion criteria in the baseline period, 14\% (2658 patients) were categorized as high-risk (immunocompromised) with $16 \%$ (3449 out of 25,110 patients) and 14\% (3612 out of 25,110 patients) identified as high-risk during the interim and follow-up periods, respectively.

Age and sex of high-risk adults age 19-64 for each observation period are provided in Table 3. Characteristics of high-risk patients age 19-64 years are presented overall and by clinic for each observation period in the Additional file 1: Tables S5-S7. Overall, patient age, sex, race and insurance status were similar across the 3 time periods; patient mean age was 50 years, $58-61 \%$ of patients were female, $78-82 \%$ were Caucasian, and nearly two-thirds were commercially insured. Generalized malignancy (48 to 62\%), iatrogenic immunosuppression (15 to $30 \%$ ), and chronic renal failure (12 to $13 \%$ )

Table 3 Demographic characteristics for adults populations studied, stratified by observation period

\begin{tabular}{|c|c|c|c|c|c|c|c|c|c|c|c|c|}
\hline & \multicolumn{3}{|c|}{$\begin{array}{l}\text { At-risk adults } \\
\text { aged 19-64 years }\end{array}$} & \multicolumn{3}{|c|}{$\begin{array}{l}\text { High-Risk Adults } \\
\text { Age 19-64 Years }\end{array}$} & \multicolumn{3}{|c|}{$\begin{array}{l}\text { Immunocompetent } \\
\text { adults aged } 65+\end{array}$} & \multicolumn{3}{|c|}{$\begin{array}{l}\text { Immunocompromised } \\
\text { Adults Age } 65+\end{array}$} \\
\hline & $\begin{array}{l}\text { Aug } 2013- \\
\text { Jul } 2014 \\
\text { (Baseline } \\
\text { Period) } \\
N=16,193\end{array}$ & $\begin{array}{l}\text { May } 2015 \text { - } \\
\text { Apr } 2016 \\
\text { (Interim } \\
\text { Period) } \\
N=18,423\end{array}$ & $\begin{array}{l}\text { May 2016- } \\
\text { Jul } 2017 \\
\text { (Follow-up } \\
\text { Period) } \\
N=21,498\end{array}$ & $\begin{array}{l}\text { Aug } 2013 \text { - } \\
\text { Jul } 2014 \\
\text { (Baseline } \\
\text { Period) } \\
N=2658\end{array}$ & $\begin{array}{l}\text { May } 2015- \\
\text { Apr } 2016 \\
\text { (Interim } \\
\text { Period) } \\
N=3449\end{array}$ & $\begin{array}{l}\text { May } 2016- \\
\text { Jul } 2017 \\
\text { (Follow-up } \\
\text { period) } \\
N=3612\end{array}$ & $\begin{array}{l}\text { Aug } 2013- \\
\text { Jul } 2014 \\
\text { (Baseline } \\
\text { Period) } \\
N=9480\end{array}$ & $\begin{array}{l}\text { May } 2015- \\
\text { Apr } 2016 \\
\text { (Interim } \\
\text { Period) } \\
N=11,318\end{array}$ & $\begin{array}{l}\text { May } 2016 \text { - } \\
\text { Jul } 2017 \\
\text { (Follow-up } \\
\text { Period) } \\
N=13,341\end{array}$ & $\begin{array}{l}\text { Aug } 2013- \\
\text { Jul } 2014 \\
\text { (Baseline } \\
\text { Period) } \\
N=2577\end{array}$ & $\begin{array}{l}\text { May } 2015- \\
\text { Apr } 2016 \\
\text { (Interim } \\
\text { Period) } \\
N=3200\end{array}$ & $\begin{array}{l}\text { May } 2016- \\
\text { Jul } 2017 \\
\text { (Follow-up } \\
\text { Period) } \\
N=3348\end{array}$ \\
\hline \multicolumn{13}{|l|}{ Age } \\
\hline $\begin{array}{l}\text { Median } \\
\text { age }\end{array}$ & 45 & 45 & 45 & 53 & 53 & 53 & 72 & 71 & 71 & 74 & 74 & 73 \\
\hline $\begin{array}{l}\text { Mean } \\
\text { age } \\
(\mathrm{SD})\end{array}$ & $\begin{array}{l}44.13 \\
(12.70)\end{array}$ & $\begin{array}{l}44.06 \\
(12.73)\end{array}$ & $\begin{array}{l}43.87 \\
(12.79)\end{array}$ & $\begin{array}{l}50.28 \\
(10.99)\end{array}$ & $\begin{array}{l}50.15 \\
(11.08)\end{array}$ & $\begin{array}{l}49.75 \\
(11.26)\end{array}$ & $\begin{array}{l}73.49 \\
(7.19)\end{array}$ & $\begin{array}{l}73.19 \\
(7.06)\end{array}$ & $\begin{array}{l}73.13 \\
(7.02)\end{array}$ & $\begin{array}{l}75.11 \\
(7.61)\end{array}$ & $\begin{array}{l}74.81 \\
(7.30)\end{array}$ & $\begin{array}{l}74.60 \\
(7.21)\end{array}$ \\
\hline \multicolumn{13}{|l|}{ Sex n (\%) } \\
\hline Male & $\begin{array}{l}7327 \\
(45.25)\end{array}$ & $\begin{array}{l}8290 \\
(45.00)\end{array}$ & $\begin{array}{l}9733 \\
(45.27)\end{array}$ & $\begin{array}{l}1111 \\
(41.80)\end{array}$ & $\begin{array}{l}1411 \\
(40.91)\end{array}$ & $\begin{array}{l}1402 \\
(38.82)\end{array}$ & $\begin{array}{l}3698 \\
(39.01)\end{array}$ & $\begin{array}{l}4555 \\
(40.25)\end{array}$ & $\begin{array}{l}5353 \\
(40.12)\end{array}$ & $\begin{array}{l}1308 \\
(50.76)\end{array}$ & $\begin{array}{l}1596 \\
(49.88)\end{array}$ & $\begin{array}{l}1654 \\
(49.40)\end{array}$ \\
\hline Female & $\begin{array}{l}8866 \\
(54.75)\end{array}$ & $\begin{array}{l}10,133 \\
(55.00)\end{array}$ & $\begin{array}{l}11,765 \\
(54.73)\end{array}$ & $\begin{array}{l}1547 \\
(58.20)\end{array}$ & $\begin{array}{l}2038 \\
(59.09)\end{array}$ & $\begin{array}{l}2210 \\
(61.18)\end{array}$ & $\begin{array}{l}5782 \\
(60.99)\end{array}$ & $\begin{array}{l}6763 \\
(59.75)\end{array}$ & $\begin{array}{l}7988 \\
(59.88)\end{array}$ & $\begin{array}{l}1269 \\
(49.24)\end{array}$ & $\begin{array}{l}1604 \\
(50.13)\end{array}$ & $\begin{array}{l}1694 \\
(50.60)\end{array}$ \\
\hline
\end{tabular}


were the 3 most common high-risk conditions across the observation periods.

In each observation period, patients in the IM Group C clinics were 2-3 years older on average than patients in FM A and B clinics. IM Group C clinics also had a higher proportion of patients with race classified as unknown, and a higher proportion of Medicare insured and chronic renal failure patients compared to FM A and B clinics. No significant differences in sex distribution were observed between clinics in the 3 observation periods.

Vaccination rates in the high-risk 19-64 years old population measured whether adults were up-to-date with the appropriate number of doses (PCV13, PPSV23 or both) by the end of the observation period. The unadjusted vaccination rate in high-risk patients 19-64 years old overall improved from $9.4 \%$ in the baseline period to 12.4 and $21.1 \%$ in the interim and follow up periods respectively $(P<0.001)$ (Additional file 4: Figure S3). While the vaccination rate improved in each clinic over the 3 observation periods, vaccination rates were significantly lower in the intervention clinics (FM Group A and FM Group B) than the IM Group C comparison clinics in all time periods. Rates improved notably for PCV13 in each clinic, increasing from approximately $2 \%$ in the baseline period to $20 \%$ or higher in the follow up period (Table 4).

In DD analyses, controlling for demographic and clinical characteristics, implementing the BPA with or without workflow redesign did not significantly affect vaccination rates from baseline to follow up (difference relative to health maintenance notifications only in the IM Group C comparison clinics: -2 percentage points, $P=0.365$ for FM Group B; -1 percentage point, $P=$ 0.704 for FM Group A) (Additional file 5: Figure S4).

\section{Vaccination rates in immunocompetent adults aged $65+$}

Of 12,057 individuals aged $65+$ who met study inclusion criteria during the baseline period, 9480 (79\%) were considered immunocompetent, with 11,318 of 14,518 (78\%) and 13,341 of 16,689 (80\%) identified as immunocompetent in the interim and follow-up periods, respectively.

Age and sex of immunocompetent adults aged 65+ for each observation period are provided in Table 1. Characteristics of immunocompetent adults aged $65+$ are presented overall and by clinic for each observation period in the Additional file 1: Tables S8-S10. Overall, patient age, sex, race and insurance status were similar across the 3 time periods; patient mean age was 73 years, $60 \%$

Table 4 Vaccination rates for PCV13 and PPSV23 overall and by intervention group within the adult populations studied

\begin{tabular}{|c|c|c|c|c|c|c|c|c|c|c|c|c|}
\hline \multirow[t]{2}{*}{ Vaccination Rates } & \multicolumn{4}{|c|}{ Family Med Clinics (A) } & \multicolumn{4}{|c|}{ Family Med Clinics (B) } & \multicolumn{4}{|c|}{ Internal Med Clinics (C) } \\
\hline & Baseline & Interim & Follow-up & $p$-value & Baseline & Interim & Follow-up & $p$-value & Baseline & Interim & Follow-up & $p$-value \\
\hline \multicolumn{13}{|c|}{ High risk adults aged 19-64 years } \\
\hline PCV13 eligible & 691 & 961 & 994 & & 732 & 982 & 1088 & & 1030 & 1369 & 1308 & \\
\hline Vaccinated (n) & 14 & 117 & 199 & $<.0001$ & 17 & 135 & 261 & $<.0001$ & 30 & 247 & 363 & $<.0001$ \\
\hline Vaccinated (\%) & 2.03 & 12.17 & 20.02 & & 2.32 & 13.75 & 23.99 & & 2.91 & 18.04 & 27.75 & \\
\hline PPSV23 eligible & 734 & 1000 & 1048 & & 785 & 1024 & 1139 & & 1125 & 1395 & 1393 & \\
\hline Vaccinated (n) & 146 & 278 & 302 & $<.0001$ & 182 & 260 & 351 & 0.0004 & 317 & 427 & 491 & 0.0005 \\
\hline Vaccinated (\%) & 19.89 & 27.80 & 28.82 & & 23.18 & 25.39 & 30.82 & & 28.18 & 30.61 & 35.25 & \\
\hline \multicolumn{13}{|c|}{ Immunocompetent adults aged $65+$} \\
\hline PCV13 eligible & & 2429 & 2807 & & & 2966 & 3724 & & & 5403 & 5530 & $<.0001$ \\
\hline Vaccinated (n) & NA & 997 & 1677 & & & 971 & 2177 & & & 2842 & 3778 & \\
\hline Vaccinated (\%) & NA & 41.05 & 59.74 & & & 32.74 & 58.46 & & & 52.60 & 68.32 & \\
\hline PPSV23 eligible & 2162 & 1796 & 2167 & & 2519 & 2360 & 2683 & & 4799 & 3519 & 4687 & \\
\hline Vaccinated (n) & 1368 & 981 & 1219 & $<.0001$ & 1356 & 1196 & 1301 & 0.001 & 3253 & 1999 & 3017 & $<.0001$ \\
\hline Vaccinated (\%) & 63.27 & 54.62 & 56.25 & & 53.83 & 50.68 & 48.49 & & 67.78 & 56.81 & 64.37 & \\
\hline \multicolumn{13}{|c|}{ Immunocompromised Adults Aged 65+ } \\
\hline PCV13 eligible & 453 & 578 & 600 & & 452 & 619 & 684 & & 1374 & 1878 & 1777 & \\
\hline Vaccinated (n) & 2 & 248 & 416 & $<.0001$ & 4 & 239 & 440 & $<.0001$ & 9 & 1091 & 1323 & $<.0001$ \\
\hline Vaccinated (\%) & 0.44 & 42.91 & 69.33 & & 0.88 & 38.61 & 64.33 & & 0.66 & 58.09 & 74.45 & \\
\hline PPSV23 eligible & 516 & 589 & 637 & & 512 & 642 & 742 & & 1549 & 1894 & 1907 & \\
\hline Vaccinated (n) & 369 & 391 & 434 & 0.180 & 327 & 416 & 492 & 0.6559 & 1144 & 1303 & 1397 & 0.0011 \\
\hline Vaccinated (\%) & 71.51 & 66.38 & 68.13 & & 63.87 & 64.8 & 66.31 & & 73.85 & 68.8 & 73.26 & \\
\hline
\end{tabular}


of patients were female, $80 \%$ were Caucasian, and $82 \%$ were insured by Medicare. Diabetes (25\%), chronic heart disease (21\%) and chronic lung disease (20\%), were the 3 most common at-risk conditions across the observation periods.

In each observation period, patients in the IM Group $C$ clinics were 1-2 years older on average than patients in FM A and B clinics. IM Group C clinics also had a higher proportion of Medicare-insured patients compared to FM A and B clinics, and a higher proportion of patients with chronic heart and lung disease. FM Group $\mathrm{B}$ clinics had a higher proportion of Hispanic patients than FM Group A or IM Group C clinics in each observation period.

Vaccination rates in immunocompetent adults aged $65+$ measured whether adults were up-to-date with PPSV23 vaccination by the end of the baseline period, and whether adults were up-to-date with the appropriate number of doses (PCV13, PPSV23 or both) by the end of the interim and follow-up periods based on changes in ACIP pneumococcal guidelines during that time. The unadjusted vaccination rate overall across all clinics decreased from $63.1 \%$ in the baseline period to $43.6 \%$ in the interim period, but rebounded to $60.5 \%$ in the follow-up period (Additional file 6: Figure S5). All clinic groups saw a decrease in PPSV23 vaccination rates in the interim period, reflecting the guideline change, with significant increases in PCV13 vaccination during both the interim and follow-up periods (Table 4).

In DD analyses, controlling for demographic and clinical characteristics, clinics that implemented the BPA with workflow redesign (FM Group A) saw a modest decrease in the vaccination rates from baseline to followup relative to IM Group $C$ clinics, but the reduction was not statistically significant (coef $-0.01 ; P=0.664$ ). Implementing the BPA (FM Group B) increased the vaccination rate 6 percentage points from baseline to followup (coef $0.06 ; P=0.001$ ) relative to the IM Group $C$ clinics. (Additional file 7: Figure S6).

\section{Vaccination rates in immunocompromised adults aged \\ $65+$}

Of 12,057 adults aged 65+ who met study inclusion criteria during the baseline period, 2577 (21\%) were considered immunocompromised. As with the immunocompetent cohort aged $65+$, the number of patients identified increased with each time period with 3200 (22\%) included in the interim period and 3348 (20\%) in the follow-up period.

Age and sex of immunocompromised adults aged 65+ for each observation period are provided in Table 1. Characteristics of immunocompromised adults age 65 years and older are presented overall and by clinic for each observation period in the Additional file 1: Tables S11-S13. Overall, patient age, sex, race and insurance status were similar across the 3 time periods; patient mean age was 75 years, $50 \%$ of patients were female, $86 \%$ were Caucasian, and $87 \%$ were insured by Medicare. Generalized malignancy (62-74\%), chronic renal failure (14-16\%), solid organ transplant (10-12\%) and iatrogenic suppression (9-19\%), were the 4 most common at-risk conditions across the observation periods.

In each observation period, patients in the IM Group $\mathrm{C}$ clinics were approximately 1 year older on average than patients in FM A and B clinics. FM Group B clinics had a higher proportion of Hispanic patients than FM Group A or IM Group C clinics. There were no significant differences in the most common immunosuppressive conditions between clinics in any of the 3 observation periods.

Vaccination rates in this population measured whether adults were up-to-date with the appropriate number of doses (PCV13, PPSV23 or both) by the end of the observation period. The unadjusted vaccination rate in highrisk patients aged $65+$ improved from $12.0 \%$ in the baseline period to 40.9 and $60.0 \%$ in the interim and follow up periods respectively $(P<0.001)$ (Additional file 8: Figure S7). While there was no difference in the vaccination rate between clinics in the baseline period, vaccination rates in the intervention FM A and B clinics were significantly lower than in the IM Group C comparison clinics in the interim and follow up periods. Rates improved significantly for PCV13 in each clinic, increasing from $<1 \%$ in the baseline period to $64 \%$ or higher in the follow up period (Table 4).

In DD analyses, controlling for demographic and clinical characteristics, the adjusted vaccination rates improved substantially from baseline to follow-up in all clinic groups. However, the increase in vaccination rates for clinics implementing both the BPA and workflow redesign (FM Group A) was 6 percentage points less (coef - 0.06; $P=0.041$ ) than in the IM Group C comparison clinics. Similarly, the increase in vaccination rates in clinics implementing only the BPA (FM Group B) was 11 percentage points lower (coef $-0.11 ; P<0.001$ ) than in the IM comparison clinics (Additional file 9: Figure S8).

\section{Series completion in adults aged $65+$}

A total of 1710 immunocompetent adults aged 65+, with documentation of a first pneumococcal vaccination with either PCV13 or PPSV23 during the baseline period, were included in the analysis of series completion. Of these, 516 patients $(30.2 \%)$ were administered the second pneumococcal dose within 6-15 months after the first dose. Series completion was examined by the presence of workflow redesign and/or clinical decision support tools in place at the time the second dose was due. The unadjusted proportion of patients who completed the series ranged from $17 \%$ in patients who received the 
second dose in clinics who had undertaken workflow redesign but before clinical decision support tools were implemented, to 35.2 and $35.6 \%$ in clinics with health maintenance notifications with and without the BPA, respectively $(P<0.001)$ (Additional file 10: Figure S9).

In adjusted analyses, patients attending a clinic that implemented the BPA plus health maintenance notifications and workflow redesign (all 3 interventions) had 1.55 times higher odds (Odds ratio (OR) 1.55; $P=0.014,95 \%$ CI $1.09,2.19)$ to receive the second pneumococcal dose than patients attending a usual practice clinic (i.e., no intervention) (Table 5). Patients attending a clinic that had implemented both the BPA and health maintenance reminders (OR 1.48; $P=0.019,95 \%$ CI $1.07,2.05)$, or just the health maintenance reminders (OR 1.83; $P<0.0001,95 \%$ CI 1.39 , $2.40)$ were also more likely to receive the second vaccination to complete the series. Patients attending clinics that undertook workflow redesign but before implementation of the BPA or health maintenance tools were less likely to have completed the series compared with patients attending a usual practice clinic (OR 0.53; $P=0.005,95 \%$ CI 0.34, 0.82). Results from the tree level mixed effects logistics regression were consistent with the results from the two level regression.

\section{Discussion}

This vaccination quality improvement study assessed the association between implementing a pneumococcal BPA tool reflecting current guidelines with and without workflow redesign on pneumococcal vaccination rates in primary care clinics affiliated with an academic medical center. The study did not consistently find a positive association between the implementation of this tool and vaccination rates in a primary care setting in each of the adult cohorts studied. Of the four groups for whom pneumococcal vaccination is recommended, use of a BPA were associated with improved vaccination rates in at-risk adults aged 19-64 years and immunocompromised adults aged $65+$ when compared with clinics who implemented health maintenance notifications only.

In at-risk adults aged 19-64 years, clinics implementing the BPA with or without workflow redesign had a greater improvement in pneumococcal vaccination rates than comparison clinics who implemented health maintenance notifications only. No difference was observed

Table 5 Likelihood of Completing the Pneumococcal Vaccination Series by Interventions in Immunocompetent Adults aged 65+ (Mixed effects logistic regression)

\begin{tabular}{|c|c|c|c|c|}
\hline & OR & $p$-value & \multicolumn{2}{|c|}{ 95\% Conf. Interval } \\
\hline \multicolumn{5}{|l|}{ Intervention } \\
\hline \multicolumn{5}{|l|}{ Current practice (ref) } \\
\hline HM Only & 1.83 & $<.0001$ & 1.39 & 2.40 \\
\hline Revised BPA + HM & 1.48 & 0.019 & 1.07 & 2.05 \\
\hline Workflow redesign only & 0.53 & 0.005 & 0.34 & 0.82 \\
\hline Revised BPA + HM + Workflow redesign & 1.55 & 0.014 & 1.09 & 2.19 \\
\hline \multicolumn{5}{|l|}{ Age at time of first dose (ref 65 years) } \\
\hline $66-75$ & 0.57 & $<.0001$ & 0.44 & 0.73 \\
\hline$>75$ & 0.34 & $<.0001$ & 0.24 & 0.48 \\
\hline \multicolumn{5}{|l|}{ Gender (ref male) } \\
\hline Female & 1.06 & 0.578 & 0.85 & 1.33 \\
\hline \multicolumn{5}{|l|}{ Insurance Status (ref Medicare) } \\
\hline Medicaid & 1.27 & 0.642 & 0.46 & 3.51 \\
\hline Commercial & 1.43 & 0.007 & 1.10 & 1.85 \\
\hline Uninsured & 1.29 & 0.481 & 0.64 & 2.58 \\
\hline Other & 2.07 & 0.617 & 0.12 & 36.07 \\
\hline Charlson Comorbidity Index & 1.00 & 0.914 & 0.94 & 1.08 \\
\hline Chronic heart disease & 0.64 & 0.011 & 0.45 & 0.90 \\
\hline \multicolumn{5}{|l|}{ Pre-index factors } \\
\hline Prior Pneumonia-Related Diagnosis & 0.97 & 0.894 & 0.63 & 1.50 \\
\hline Number of office visits 12 -mos pre-index & 1.02 & 0.345 & 0.98 & 1.07 \\
\hline
\end{tabular}


in the age 19-64 high-risk group between clinics with the BPA or workflow redesign versus the comparison clinics. Similarly, in the aged $65+$ immunocompetent cohort, no difference was seen between clinics that implemented both the BPA and workflow redesign versus comparison clinics. However, clinics that implemented just the BPA (FM Group B) had a greater improvement in vaccination rate (coef $0.06 ; P=0.001$ ) relative to the IM Group C clinics.

In immunocompromised adults aged $65+$, clinics that implemented the BPA with or without workflow redesign experienced a smaller improvement in vaccination rates relative to the comparison clinics. However, over the study period there was a 4-fold improvement in vaccination rates for all clinic groups (e.g. from 9 to $10 \%$ at baseline to $46-50 \%$ at follow-up), driven by a substantial uptake of PCV13 vaccination.

The second objective of the study was to longitudinally assess series completion over time in a cohort of immunocompetent older adults. This analysis found that patients attending clinics where the clinical decision support tools were implemented at the time their second dose was due, including BPA with health maintenance or health maintenance notifications alone, were more likely to receive the second vaccination than patients attending clinics without clinical decision support tools. Patients in the clinics that had undertaken workflow changes but before implementing the BPA and health maintenance tools were less likely to have received the second dose than those in usual care clinics. This finding was unexpected given the clinics' focus on improving pneumococcal vaccination rates, but may reflect challenges of implementing new workflows during the period following guideline updates for this population.

Clinical decision support tools can help health care providers identify patients who are eligible for vaccinations. [6] For instance, the impact of an electronic medical record-based BPA that alerted providers to administer influenza and pneumococcal vaccines to adult patients taking immunosuppressive drugs was evaluated at the Geisinger Health System Rheumatology Department. Following BPA implementation, the pneumococcal vaccination rate in immunocompromised adults doubled from 19 to $41 \%(p<0.001)$. [7]

On the other hand, numerous barriers to clinical decision support implementation have been also identified, which may help explain the mixed findings in this study. Barriers include competing clinical demands, reliability of information, complexity, physician attitudes, too many unwanted alerts, poor alert timing, and lack of acceptance of CDS tools. [8, 11, 12]

While $U$ of $U$ Health clinicians voiced a need for assistance with implementing pneumococcal vaccination guidelines, the guidelines and the supporting logic are complex. Even with extensive input from clinicians and implementation oversight by the $U$ of $U$ Health clinical decision support committee, it was challenging to systematically and accurately capture conditions that make patients candidates for pneumococcal vaccination in the BPA. This was particularly true for patients immunosuppressed at the time of the analyses and those with chronic lung disease or heart disease. While the $U$ of $U$ Health clinical decision support development team was successful in integrating a two-way, real-time information exchange with the state immunization registry to help improve accuracy and completeness of vaccination history data, numerous reports of actual or perceived "misfires" were made by clinicians after implementation, which may have reduced provider confidence in the information.

A positive association between the BPA in at-risk adults age 19-64 years and series completion in immunocompetent adults aged $65+$ was identified. In these scenarios, providers only needed to provide a single PPSV-23 dose to atrisk adults age 19-64 and provide the second dose in the recommended series for adults aged $65+$. In a post-hoc analysis, vaccination rates were further explored by select comorbidities. Patients with diabetes had higher vaccination rates and greater improvement from baseline to follow-up in the clinics with the BPA (FM Group A \& B) versus comparison clinics (IM Group C). This same pattern was not observed for chronic lung and heart disease. This observed pattern may be due to greater complexity in interpreting which patients fit the definition of at-risk for heart and lung disease than for diabetes. Overall, these findings suggest that the BPA was associated with an increase in pneumococcal vaccination rates when applying the guidelines in less complex patient care scenarios.

Much of the gain in pneumococcal vaccination rates in high-risk adults age 19-64 years and immunocompromised adults aged 65+ was due to the large uptake in PCV13 vaccinations. While no system-wide efforts were in place to increase PCV13 vaccinations at $\mathrm{U}$ of $\mathrm{U}$ Health,this gain corresponds with the timing of guideline changes that added PCV13 to the aged 65+ immunocompetent patient recommendations. This guideline update may have universally increased consumer and provider awareness and overshadowed the effect of the BPA and workflow redesign on vaccination rates for all age and risk groups for which PCV-13 is recommended, even though recommendations for sequential PCV13PPSV23 vaccination have been in place for high-risk/immunocompromised adults age 19 and above since 2012.

Finally, health maintenance reminders were implemented system-wide at $U$ of $U$ Health in the followup period, including in the IM Group C comparison clinics. Based on other, recent experience at $\mathrm{U}$ of $\mathrm{U}$ 
Health, it is possible that health maintenance reminders may be better received and more likely to be followed than BPAs. This study was not specifically designed to answer that question; however, it will be the topic of future research.

\section{Limitations}

This study has a number of limitations that could affect results. First, this was an observational study of a planned quality improvement initiative, and thus clinics were not randomized to interventions. While an internal comparison group was included and a statistical method employed to help control for underlying trends, there may be other factors that affected vaccination rates which were not controlled for.

Vaccination status was based upon documentation of vaccination administration and patient reported history in the electronic medical record by providers/ clinic staff. While documentation of $U$ of $U$ Health administered vaccines should be complete, patient recall of pneumococcal vaccination given outside $U$ of $\mathrm{U}$ Health may be inaccurate. Access to USIIS data should help to reduce recall bias, however documentation in USIIS is only mandatory for adult vaccinations administered in pharmacies in Utah. Thus, this lack of complete and accurate vaccination history in $U$ of $U$ Health systems may have introduced misclassification of pneumococcal vaccination status leading to an overall underestimation of vaccination rates.

Vaccination rates were calculated using the total number of eligible adults age 19-64 years who attended clinics, rather than the total eligible population within the catchment area of each clinic group. Whilst the denominator could be the total population within a defined geographical area, these data were not collected as part of this study.

ICD-9/10 codes were used to identify vaccine eligible conditions. While the presence of 2 codes for a given condition on separate days was required to avoid misclassifying patients, diagnosis codes often lack the specificity to accurately identify at-risk, immunocompetent versus high-risk and currently immunocompromised cohorts. To gain provider confidence, the BPA was turned off for approximately 2 weeks during the first month of implementation while adjustments were made to atrisk/high-risk condition groupers and clinical decision support logic rules. Thus, reporting of vaccine eligible conditions reflects these modifications over time. Since the time the BPA was turned off represents only a small portion of the total follow-up time, it is unlikely that vaccination rates or series completion was significantly affected.

Finally, patient clinic assignment was based on the location of the patient's last office visit during the observation period for the analysis of vaccination rates and at the time the second dose was due for the series completion evaluation. Patients in the $\mathrm{U}$ of $\mathrm{U}$ Health tend to visit the same clinic on an ongoing basis, but the assigned clinic may not have been the patient's primary clinic leading to misclassification of exposure. Since the same method for assigning clinic was used for all patients, it is unlikely that this bias favors one clinic group over the other.

\section{Conclusion}

A pneumococcal BPA tool that reflects current guidelines implemented with and without workflow redesign improved vaccination rates for at-risk adults age 19-64 years and increased the likelihood of adults aged $65+$ receiving their second dose to complete the recommended 2-dose series. However, in other adult patient groups, the BPA was not consistently associated with improvements in pneumococcal vaccination rates. Additional research is warranted to identify barriers to acting upon the BPA and to assess the impact of health maintenance reminders as an alternative approach to promoting adherence to pneumococcal vaccination guidelines.

\section{Additional files}

Additional file 1: Table S1. Recommended immunization and intervals, by risk and age groups, for persons with indications to receive pneumococcal immunization. Table S2. Demographic Characteristics for At-Risk Adults Age 19-64 Years Overall and Stratified by Intervention Group - Aug 2013 - Jul 2014, Baseline Period. Table S3. Demographic Characteristics for At-Risk Adults Age 19-64 Years Overall and Stratified by Intervention Group - May 2015 - Apr 2016 (Interim Period). Table S4. Demographic Characteristics for At-Risk Adults Age 19-64 Years Overall and Stratified by Intervention Group - May 2016 - Jul 2017 (Follow-up Period). Table S5. Demographic Characteristics for High-Risk Adults Age 19-64 Years Overall and Stratified by Intervention Group - Aug 2013 - Jul 2014 (Baseline Period). Table S6. Demographic Characteristics for HighRisk Adults Age 19-64 Years Overall and Stratified by Intervention Group May 2015 - Apr 2016 (Interim Period). Table S7. Demographic Characteristics for High-Risk Adults Age 19-64 Years Overall and Stratified by Intervention Group - May 2016 - Jul 2017 (Follow-up Period). Table S8. Demographic Characteristics for Immunocompetent Adults Aged 65+ Overall and Stratified by Intervention Group - Aug 2013 - Jul 2014 (Baseline Period). Table S9. Demographic Characteristics for Immunocompetent Adults Aged 65+ Overall and Stratified by Intervention Group - May 2015 - Apr 2016 (Interim Period). Table S10. Demographic Characteristics for Immunocompetent Adults Aged 65+ Overall and Stratified by Intervention Group - May 2016 - Jul 2017 (Follow-up Period). Table S11. Demographic Characteristics for Immunocompromised Adults Aged 65+ Overall and Stratified by Intervention Group - Aug 2013 - Jul 2014 (Baseline Period). Table S12. Demographic Characteristics for Immunocompromised Adults Aged 65+ Overall and Stratified by Intervention Group - May 2015 - Apr 2016 (Interim Period). Table S13. Demographic Characteristics for Immunocompromised Adults Aged 65+ Overall and Stratified by Intervention Group - May 2016 - Jul 2017 (Follow-up Period). (DOCX $132 \mathrm{~kb}$ )

Additional file 2: Figure S1. Vaccination Rates for At-Risk Adults Age 19-64 Years by Clinic Group and Overall. Description: The vaccination 
rates of at-risk adults age 19-64 years by clinic group and overall over the three time periods studied. (DOCX $59 \mathrm{~kb}$ )

Additional file 3: Figure S2. Intervention Effect on Pneumococcal Vaccination Rates: Implementing BPA and/or Workflow Redesign versus Health Maintenance Notifications - At-Risk Patients age 19-64 Years. Description: Pneumococcal vaccination rates in at-risk patients age 19-64 years in clinics implementing BPA and/or workflow redesigns, versus health maintenance notifications. (DOCX $31 \mathrm{~kb}$ )

Additional file 4: Figure S3. Vaccination Rates for High-Risk Adults Age 19-64 Years by Clinic Group and Overall. Description: The vaccination rates of high-risk adults age 19-64 years by clinic group and overall over the three time periods studied. (DOCX $60 \mathrm{~kb}$ )

Additional file 5: Figure S4. Intervention Effect on Pneumococcal Vaccination Rates: Implementing BPA and/or Workflow Redesign versus Health Maintenance Notifications - High-Risk Patients age 19-64 Years. Description: Pneumococcal vaccination rates in high-risk patients age 19-64 years in clinics implementing BPA and/or workflow redesigns, versus health maintenance notifications. (DOCX $30 \mathrm{~kb}$ )

Additional file 6: Figure S5. Vaccination Rates for Immunocompetent Adults Aged $65+$ by Clinic Group and Overall. Description: The vaccination rates of immunocompetent adults age $65+$ years by clinic group and overall over the three time periods studied. (DOCX $54 \mathrm{~kb}$ )

Additional file 7: Figure S6. Intervention Effect on Pneumococcal Vaccination Rates: Implementing Workflow Redesign and/or BPA Versus Comparison Clinics based on Difference in Difference Analyses Immunocompetent Patients aged 65+. Description: Pneumococcal vaccination rates in immunocompetent patients age $65+$ years in clinics implementing workflow redesign and/or BPA, versus comparison clinics based on difference in difference analyses. (DOCX $31 \mathrm{~kb}$ )

Additional file 8: Figure S7. Vaccination Rates for Immunocompromised Adults Aged 65+ by Clinic Group and Overall. Description: The vaccination rates of immunocompromised adults age $65+$ years by clinic group and overall over the three time periods studied. (DOCX 64 kb)

Additional file 9: Figure S8. Intervention Effect on Pneumococcal Vaccination Rates: Implementing Workflow Redesign and/or BPA Versus Comparison Clinics based on Difference in Difference Analyses Immunocompromised Patients aged 65+. Description: Pneumococcal vaccination rates in immunocompromised patients aged $65+$ years in clinics implementing workflow redesign and/or BPA, versus comparison clinics based on difference in difference analyses. (DOCX $31 \mathrm{~kb}$ )

Additional file 10: Figure S9. Pneumococcal Vaccination Series Completion by Intervention Category. Description: Pneumococcal vaccination series completion by intervention category, including no intervention, and all three interventions overall. (DOCX 59 kb)

\section{Abbreviations}

ACIP: Advisory Committee on Immunization Practices; BPA: Best Practice Alert; CPT: Care delivery and billing codes; DD: Difference in differences; EDW: Enterprise Data Warehouse; FM: Family Medicine; HIV: Human immunodeficiency virus; IM: Internal Medicine; PCV13: Pneumococcal conjugate vaccine; PPSV23: Pneumococcal polysaccharide vaccine; $U$ of $U$ Health: University of Utah Health; US: United States; USIIS: Utah State Immunization Information System

\section{Acknowledgements}

The authors thank Hannah Collings, Robert Taaffe, and Paul Okhuoya, employees of Adelphi Values, for their assistance with manuscript preparation and submission.

\section{Availability of data and material}

The datasets generated and/or analyzed during the current study are not publicly available due to patient privacy/confidentiality, but are available from the corresponding author on reasonable request.

\section{Authors' contributions}

CM contributed to the conception, design or planning of the study, acquisition of the data interpretation of study results, drafting of the manuscript, and reviewing or revising the manuscript for important intellectual content. CT contributed to the conception, design or planning of the study, acquisition of the data, analysis of the data, interpretation of study results, and reviewing or revising the manuscript for important intellectual content. TP contributed to the conception, design or planning of the study, interpretation of study results, drafting of the manuscript and reviewing or revising the manuscript for important intellectual content. NWJ analysis of the data, interpretation of study results, drafting of the manuscript and reviewing or revising the manuscript for important intellectual content. MY contributed to analysis of the data, interpretation of study results, and reviewing or revising the manuscript for important intellectual content. MB contributed to the conception, design or planning of the study, and reviewing or revising the manuscript for important intellectual content. KG contributed to the conception, design or planning of the study, interpretation of study results, and reviewing or revising the manuscript for important intellectual content. LG contributed to the conception, design or planning of the study, analysis of the data, interpretation of study results and reviewing or revising the manuscript for important intellectual content. All authors read and approved the final manuscript.

\section{Funding}

Funding for this research was provided by Merck \& Co., Inc., Kenilworth, NJ, USA. The funding body contributed to design of the study, data analysis and interpretation of data. Dr. Petigara is an employee of Merck \& Co., Inc., Kenilworth, NJ, USA and contributed to the writing of the manuscript.

\section{Ethics approval and consent to participate}

This study was deemed exempt by the University of Utah Institutional Review Board (IRB \#00079432). As a result of this IRB exemption, informed consent was therefore also not required from participants for this retrospective study.

\section{Consent for publication}

Not applicable.

\section{Competing interests}

Conflict of interest statement: Dr. Petigara is an employee of Merck \& Co., Inc., Kenilworth, NJ, USA. Funding for this research was provided by Merck \& Co., Inc., Kenilworth, NJ, USA.

The authors declare that they have no competing interests.

\section{Author details}

${ }^{1}$ Department of Pharmacotherapy, University of Utah, Salt Lake City, UT, USA. ${ }^{2}$ Division of Pharmaceutical Evaluation \& Policy, University of Arkansas for Medical Sciences, 4301 W. Markham St, Little Rock, AR 72205, USA. ${ }^{3}$ Division of Pharmaceutical Outcomes and Policy, University of North Carolina at Chapel Hill, Chapel Hill, NC, USA. ${ }^{4}$ Merck \& Co., Inc, Kenilworth, NJ, USA. ${ }^{5}$ Division of Public Health, University of Utah, Salt Lake City, UT, USA. ${ }^{6}$ Department of Economics, University of Utah, Salt Lake City, UT, USA. ${ }^{7}$ University of Utah Health, Salt Lake City, UT, USA. ${ }^{8}$ Department of Family \& Preventive Medicine, University of Utah, Salt Lake City, UT, USA.

Received: 1 November 2018 Accepted: 14 June 2019 Published online: 10 July 2019

\section{References}

1. McLaughlin JM, McGinnis JJ, Tan L, Mercatante A, Fortuna J. Estimated human and economic burden of four major adult vaccine-preventable diseases in the United States, 2013. J Prim Prev. 2015:36(4):259-73.

2. Centers for Disease C, Prevention. Use of 13-valent pneumococcal conjugate vaccine and 23-valent pneumococcal polysaccharide vaccine for adults with immunocompromising conditions: recommendations of the advisory committee on immunization practices (ACIP). MMWR Morb Mortal Wkly Rep. 2012;61(40):816-9.

3. Tomczyk S, Bennett NM, Stoecker C, Gierke R, Moore MR, Whitney CG, et al. Use of 13-valent pneumococcal conjugate vaccine and 23-valent pneumococcal polysaccharide vaccine among adults aged $>/=65$ years: recommendations of the advisory committee on immunization practices (ACIP). MMWR Morb Mortal Wkly Rep. 2014;63(37):822-5.

4. Office of Disease Prevention and Health Promotion. Health people 2020 Washington, DC: Department of Health and Human Services. [Available 
from: https://www.healthypeople.gov/2020/topics-objectives/topic/ immunization-and-infectious-diseases/objectives]

5. Williams WW, Lu PJ, O'Halloran A, Kim DK, Grohskopf LA, Pilishvili T, et al. Surveillance of vaccination coverage among adult populations - United States, 2015. MMWR Surveill Summ. 2017;66(11):1-28.

6. Stockwell MS, Fiks AG. Utilizing health information technology to improve vaccine communication and coverage. Hum Vaccin Immunother. 2013;9(8): 1802-11.

7. Ledwich LJ, Harrington TM, Ayoub WT, Sartorius JA, Newman ED. Improved influenza and pneumococcal vaccination in rheumatology patients taking immunosuppressants using an electronic health record best practice alert. Arthritis Rheum. 2009;61(11):1505-10.

8. Stockwell MS, Catallozzi M, Camargo S, Ramakrishnan R, Holleran S, Findley $\mathrm{SE}$, et al. Registry-linked electronic influenza vaccine provider reminders: a cluster-crossover trial. Pediatrics. 2015;135(1):e75-82.

9. Ai C, Norton E. Interaction terms in logit and probit models. Econ Lett. 2003; 80(1):123-9.

10. Bertrand M, Duflo E, Mullainathan S. How much should we trust differencesn-differences estimates? Quart J Econ. 2004;119(1):249-75.

11. Devaraj S, Sharma S, Fausto D, Viernes S, Kharrazi H. Barriers and facilitators to clinical Decison support systems adoption: a systematic review. J Bus Admin Res. 2014;3(2):36-53.

12. Berner E. Clinical decision support systems: State of the Art. Rockville: AHRQ: 2009. June 2009. Report No.: 09-0069-EF

\section{Publisher's Note}

Springer Nature remains neutral with regard to jurisdictional claims in published maps and institutional affiliations.

Ready to submit your research? Choose BMC and benefit from:

- fast, convenient online submission

- thorough peer review by experienced researchers in your field

- rapid publication on acceptance

- support for research data, including large and complex data types

- gold Open Access which fosters wider collaboration and increased citations

- maximum visibility for your research: over $100 \mathrm{M}$ website views per year

At $\mathrm{BMC}$, research is always in progress.

Learn more biomedcentral.com/submissions 